\title{
Tumor Demarcation in Mammography Images using LBG on Probability Image
}

\author{
Dr. H.B.Kekre \\ Senior Professor, MPSTME, NMIMS \\ University, Vile-Parle (w), Mumbai- \\ 400056, India
}

\author{
Saylee M. Gharge \\ Ph.D. Scholar, MPSTME, NMIMS \\ University, Lecturer, V.E.S.I.T, \\ Mumbai-400071, India
}

\author{
Tanuja K. Sarode \\ Ph.D. Scholar, MPSTME, NMIMS \\ University , Assistant Professor, \\ TSEC, Mumbai-400050, India
}

\begin{abstract}
The ability to improve diagnostic information from medical images can be enhanced by designing computer processing algorithms that is why we proposed new algorithm to detect cancer in mammogram breast cancer images. In this paper we proposed segmentation using vector quantization technique. Here we used Linde Buzo and Gray (LBG)for segmentation of mammographic images on probability image. Initially probability of input image is calculated and displayed as a result. In second step a codebook of size 128 was generated for probability image. These code vectors were further reclustered in 8 clusters using same LBG algorithm. These 8 images were displayed as a result. This approach does not leads to over segmentation or under segmentation. For the comparison purpose we displayed results of GLCM and watershed segmentation along with this method.
\end{abstract}

\section{General Terms}

Algorithms

\section{Keywords}

Mammography, segmentation, tumor detection, LBG.

\section{INTRODUCTION}

Image segmentation is, by definition, the problem of decomposing images into regions that are semantically uniform. However, since images themselves provide only semantically poor information, image segmentation is essentially an application-oriented problem that demands either strong intervention of human experts or application specific solutions. In other words, no fully automatic, general-purpose segmentation method exists.

Breast image analysis can be performed using X-rays, magnetic resonance, nuclear medicine or ultrasound. So far the most effective and economical breast imaging modality has been X-ray mammography due to its simplicity, portability and cost effectiveness. Recent studies showed that the interpretation of the mammogram by the radiologists give high rates of false positive cases Indeed the images provided by different patients have different dynamics of intensity and present a weak contrast. Moreover the size of the significant details can be very small. Several research works have tried to develop computer aided diagnosis tools. They could help the radiologists in the interpretation of the mammograms and could be useful for an accurate diagnosis. In order to perform a semi-automated tracking of the breast cancer, it is necessary to detect the presence or absence of lesions from the mammograms[1,2].These lesions can be of various types: - Nodular opacities, clear masses with lobed edges etc. They can be benign or malignant, according to their contour (sharp or blurred)-Stellar opacities (malignant tumors); micro calcifications: small calcified structures that appear as clear points on a mammogram $[3,4]$.

For mammograms manifesting masses this corresponds to the detection of suspicious mass regions. A number of image processing methods have been proposed to perform this task. S. M. Lai et al [5] and W. Qian et al [6] have proposed using modified and weighted median filtering, respectively, to enhance the digitized image prior to object identification. D. Brzakovic et all [7] used thresholding and fuzzy pyramid linking for mass localization and classification. Other investigators have proposed using the asymmetry between the right and left breast images to determine possible mass locations. Yin et al. uses both linear and nonlinear bilateral subtraction [8] while the method by Lau et al. [9] relies on "structural asymmetry" between the two breast images. Recently Kegelmeyer [10] has reported promising results for detecting spiculated lesions based on local edge characteristics and Laws texture features [11-13].The above methods produced a true positive detection rate of approximately $90 \%$. The work we have done is to propose a segmentation process which identifies on a mammogram the opaque areas, suspect or not, present in the image using vector quantization.

Segmenting a mammographic images into homogeneous texture regions representing disparate tissue types is often a useful preprocessing step in the computer-assisted detection of breast cancer. Various segmentation techniques have been proposed based on statistically measurable features in the image [1423].Clustering algorithms, such as k-means and ISODATA, operate in an unsupervised mode and have been applied to a wide range of classification problems[24].

The choice of a particular technique depends on the application, on the nature of the images available (texture, ill-defined contours, shadows), on the primitives to be extracted (contours, straight segments, regions, shapes), number of objects need to be 
segmented, the amount of available user time, and the required accuracy of the segmentation.

We are introducing vector quantization for image Segmentation with post processing which consumed moderate time but provide good accuracy with less complexity.

\subsection{Vector Quantization}

Vector Quantization (VQ) [25-33],[52-54] is an efficient technique for data compression and has been successfully used in various applications such as index compression [24, 35]. VQ has been very popular in a variety of research fields such as speech recognition and face detection [36, 37]. VQ is also used in real time applications such as real time video-based event detection [38] and anomaly intrusion detection systems [39], image segmentation [40-43], speech data compression [44], content based image retrieval CBIR [45] and face recognition [46].

Vector Quantization (VQ) techniques employ the process of clustering. Various VQ algorithms differ from one another on the basis of the approach employed for cluster formations. VQ is a technique in which a codebook is generated for each image. A codebook is a representation of the entire image containing a definite pixel pattern which is computed according to a specific VQ algorithm.

The rest of the paper is organized as follows. Section II describes Gray level Co-occurrence Matrix, the Watershed and Linde Buzo and Gray (LBG) algorithm used for image segmentation of mammographic images. Followed by the experimental results for mammographic images are in section III and section IV concludes the work.

\section{ALGORITHMS FOR SEGMENTATION}

It is difficult, however, to compare the effectiveness of these methods because each used a unique set of digitized mammograms and the results varied between training and testing.

In this section we explain segmentation by Gray level cooccurrence matrix , basic watershed algorithm [47-51] and Linde Buzo and Gray algorithm [52-54] which we used for tumor detection

\subsection{Gray Level Co-occurrence Matrix}

Haralick [55] suggested the use of gray level co-occurrence matrices (GLCM) for definition of textural features. The values of the co-occurrence matrix elements present relative frequencies with which two neighboring pixels separated by distance d appear on the image, where one of them has gray level $i$ and other j. Such matrix is symmetric and also a function of the angular relationship between two neighboring pixels. The cooccurrences matrix can be calculated on the whole image, but by calculating it in a small window which scanning the image, the co-occurrence matrix can be associated with each pixel. By using gray level co-occurrence matrix we can extract different features like probability, entropy, energy, variance, inverse moment difference etc. For this paper we extracted entropy using gray level co-occurrence matrix and displayed here as a result in Fig.3(a) in comparison with watershed and LBG algorithms.

\subsection{Watershed algorithm}

Watershed segmentation [56] classifies pixels into regions using gradient descent on image features and analysis of weak points along region boundaries. The image feature space is treated, using a suitable mapping, as a topological surface where higher values indicate the presence of boundaries in the original image data. It uses analogy with water gradually filling low lying landscape basins. The size of the basins grow with increasing amounts of water until they spill into one another. Small basins (regions) gradually merge together into larger basins. Regions are formed by using local geometric structure to associate the image domain features with local extremes measurement. Watershed techniques produce a hierarchy of segmentations, thus the resulting segmentation has to be selected using either some prior knowledge or manually. These methods are well suited for different measurements fusion and they are less sensitive to user defined thresholds. We implemented watershed algorithm for mammographic images as mentioned in [57].Results for mammographic images are displayed in Fig3 (b).

\subsection{Proposed Algorithm}

In this proposed algorithm probability of original image is used for grouping pixels into regions and then the image of probability is formed. For further image segmentation equalized probability image is used as an input image.

The proposed technique has three steps to follow:

1. Region forming using vector quantization technique

2. Region merging using reclustering.

3. Post Processing.

\subsubsection{Probability}

For complete image we will find out probability of particular $\mathrm{i}$ th gray level which is given by:

$$
\text { Probability } \mathrm{P}(\mathrm{i})=\frac{X_{i}}{M X N}
$$

Where $X \mathrm{i}$ is number of pixels for $\mathrm{i}^{\text {th }}$ gray levels, $\mathrm{M}$ and $\mathrm{N}$ are no. of rows and columns of the image.

After calculating this we form the image which contains probability values for that particular gray level instead of gray level in the image and it is displayed as probability image as shown in Figure 2(b) for original image in Figure 2(a) and Figure 2(c) is histogram equalized probability image. 


\subsubsection{Vector Quantization}

Vector Quantization (VQ) techniques employ the process of clustering. Various VQ algorithms differ from one another on the basis of the approach employed for cluster formations. VQ is a technique in which a codebook is generated for each image. A codebook is a representation of the entire image containing a definite pixel pattern which is computed according to a specific VQ algorithm[58-60]. The image is divided into fixed sized blocks that form the training vector. The generation of the training vector is the first step to cluster formation on these training vectors clustering methods is applied and codebook is generated.

\subsubsection{1..Linde Buzo and Gray Algorithm (LBG)}

For the purpose of explaining this algorithm, we are considering two dimensional vector space as shown in Fig.1. In this algorithm centroid is computed as the first codevector $\mathrm{C} 1$ for the training set. In Fig. 1 two vectors v1 \& v2 are generated by adding constant error to the codevector. Euclidean distances of all the training vectors are computed with vectors v1 \& v2 and two clusters are formed based on nearest of v1 or v2. Procedure is repeated for these two clusters to generate four new clusters. This procedure is repeated for every new cluster until the required size of codebook is reached or specified MSE is reached.

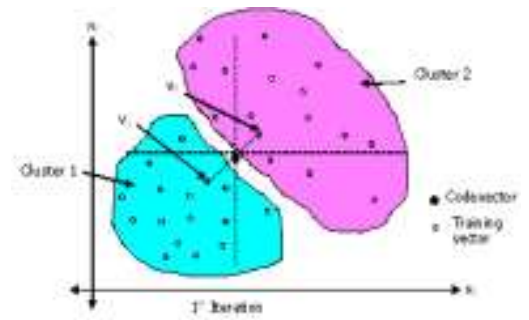

Fig.1. LBG for 2 dimensional case
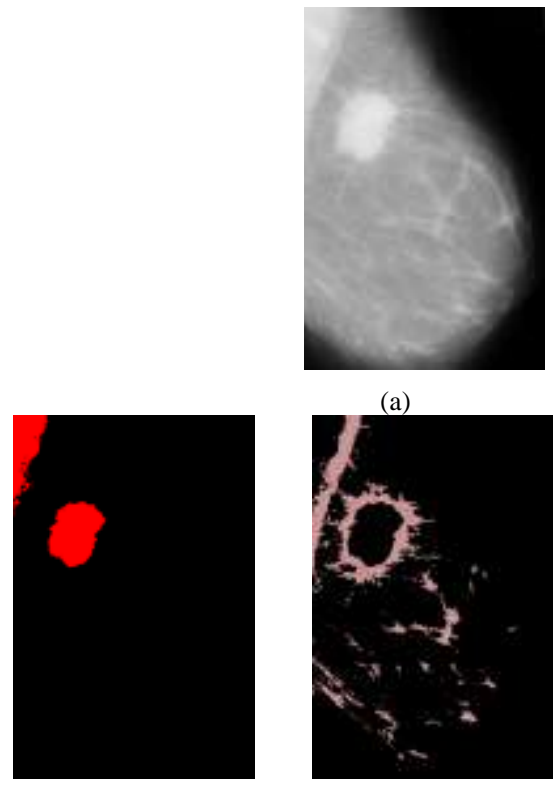

(c)

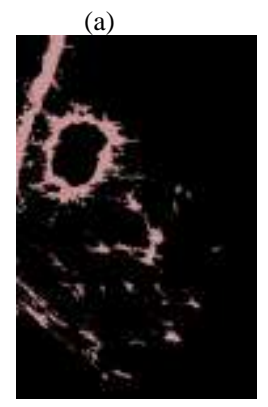

(d)
Using this algorithm initially a codebook of size 128 was generated for the given images. These code-vectors were further re-clustered in 8 clusters using same LBG algorithm this is nothing but region merging.The 8 images were constructed using one code-vector at a time. These 8 images display different segments depending on the textural property of the image. It is observed that the image constructed using first code vector displays tumor clearly.

\subsection{3..Post Processing}

If some unwanted regions are present in the segmented image then post processing is required to achieve only tumor in the final image .For that morphological operations are used .For proper boundary, canny edge detector is used .This edge map is superimposed on original image for comparison purpose as shown in Fig.4(a)-(c).

\section{RESULTS}

Mammography images from mini-mias database were used in this paper for implementation of GLCM, watershed and proposed algorithms for tumor demarcation.Fig.2(a) shows original image with tumor. Fig.2(b) indicates probability image which is formed by using original image. For this image codebook of size 128 is generated using LBG algorithm. Further these code-vectors were reclustered in 8 clusters using same algorithm. Then 8 images were constructed using one code-vector at a time as shown in Fig.2 (c)-(j). Further post processing is applied on first codevector image .Fig.3 (a) shows results for equalized entropy using gray level co-occurrence matrix.Fig.3(b)shows result for watershed algorithm and Fig.3(c) indicate result of proposed algorithm.Fig.4(a)-(c) displays superimposed image by canny edge detector on original image for image Fig.3(a)-(c) respectively for comparison purpose.
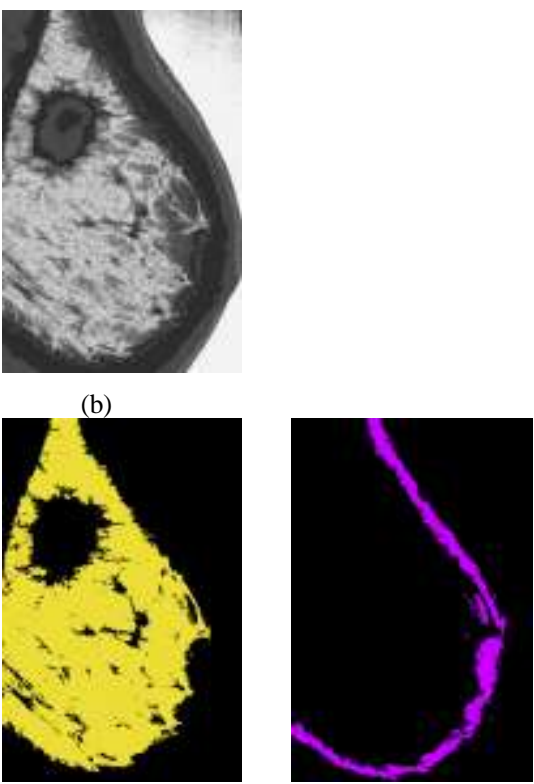

(e)

(f) 


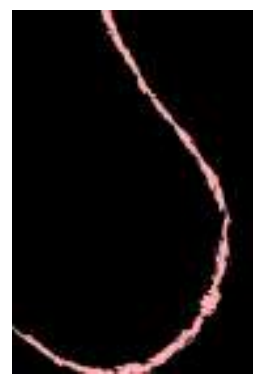

(g)

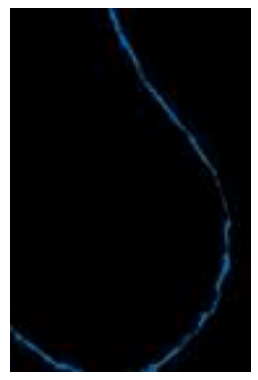

(h)

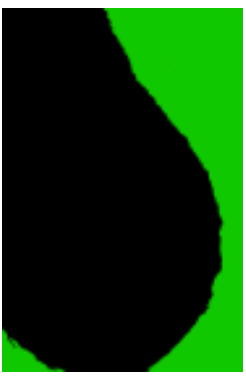

(i)

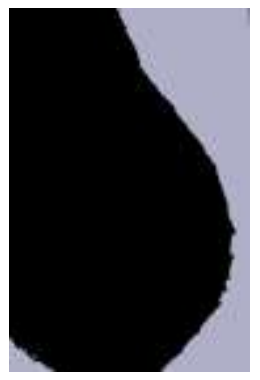

(j)

Fig. 2: (a) Original breast tumor image,(b)Equalized probability image ,(c) Image for first code-vector, (d)Image for second code-vector, (e)Image for third code-vector, (f) Image for fourth code-vector, (g) Image for fifth code-vector,

(h)Image for sixth code-vector, (i) Image for seventh code-vector, (j) Image for eighth code-vector,

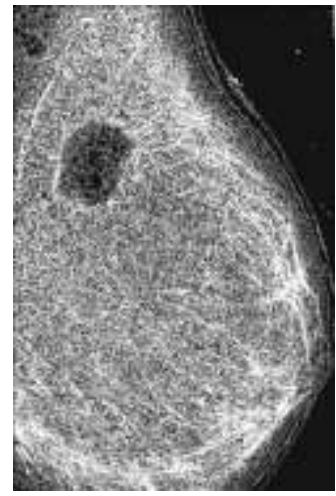

(a)

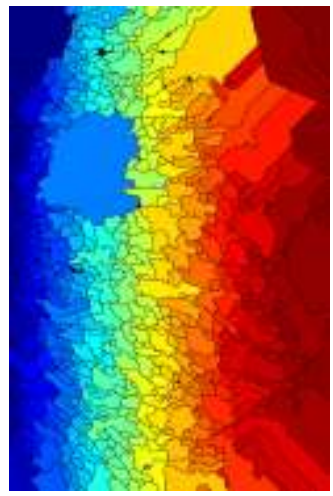

(b)

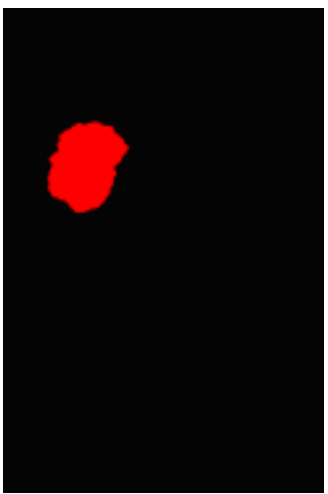

(c)

Fig.3: (a) Segmented image for entropy using GLCM, (b) Segmented image using watershed algorithm,

(c)Segmented image using proposed algorithm

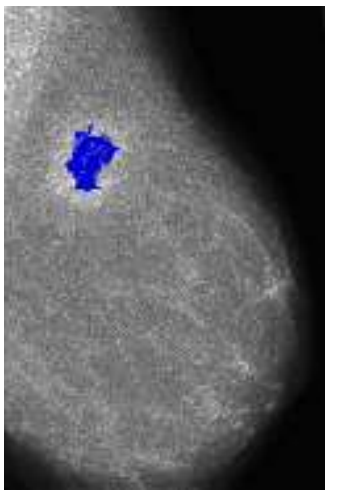

(a)

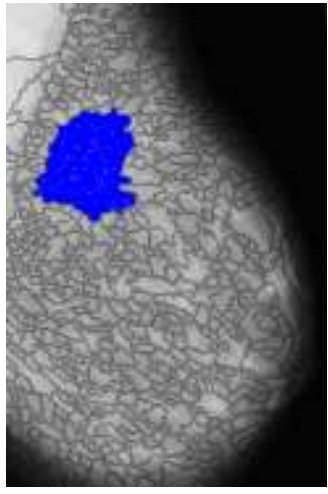

(b)

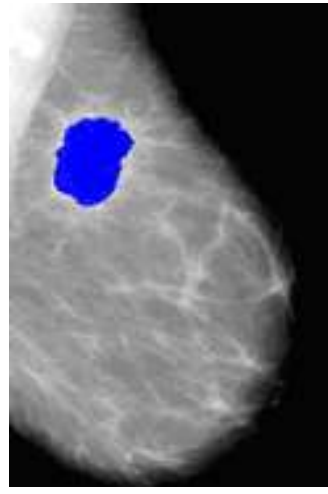

(c)

Fig. 4 : (a)-(c)Superimposed images Fig3(a)-(c) on original image fig.2(a)

\section{CONCLUSION}

In this paper vector quantization is used which is commonly used for data compression. Basically vector quantization is a clustering algorithm and can be used for texture analysis. Here the results of proposed algorithm using LBG for tumor detection in mammographic images are displayed. The results are compared with well known watershed algorithm and GLCM. Fig 2(c)-(j) shows variation in texture using LBG algorithm. It is observed that watershed and GLCM give over segmentation while LBG shows far better results for the same. Post processing improves the results further which will help in further investigation or surgery by Doctors.This approach does not lead to over segmentation or under segmentation.

\section{ACKNOWLEDGEMENT}

The authors would like to record their appreciation to Dr. Manisha Munde for her help in validating the results. 


\section{REFERENCES}

[1]E. E. Sterns, "Relation between clinical and mammographic diagnosis of breast problems and the cancer/ biopsy rate," Can. J. Surg., vol. 39, nº. 2, pp. 128-132, 1996.

[2] R. Highnam and M. Brady, Mammographic Image Analysis , Kluwer Academic Publishers, 1999. ISBN: 0-7923- 5620-9.

[3] Matthew A. Kupinski and Maryellen L. Giger, "Automated Seeded Lesion Segmentation" IEEE Transaction on medical imaging, Vol.17,No.4,August 1998.

[4] Wirth, M.A. Stapinski, A., "Segmentation of the breast region in mammograms using active contours", in Visual Communications and Image Processing, Switzerland, 2003, Vol. 5150, pp. 1995-2006.

[5] S. M. Lai, X. Li, and W. F. Bischof, " On techniques for detecting circumscribed masses in mammograms," IEEE Trans. Med. Zmag., vol. 8, no. 4, pp. 377-386, Dec. 1989.

[6] W. Qian, L. P. Clarke, M. Kallergi, and R. A. Clark, “ Treestructured nonlinear filters in digital mammography, IEEE Trans. Med. Zmag., vol.13, no. 1, pp. 25-36, Mar. 1994.

[7] D. Brzakovic, X. M. Luo, and P. 1Bzrakovic, "An approach to automated detection of tumors in mammography," IEEE Trans. Med. Imag., vol. 9, no. 3, pp. 233-241, Sept. 1990.

[8] F. F. Yin, M. L. Giger, K. Dol, C. E. Metz, R. A. Vyborny, and C. J. Schmidt, "Computerized detection of masses in digital mammograms: Analysis of bilateral subtraction images,'” Med. Phys., vol. 18, no. 5, pp. 955-963, Sept. 1991.

[9] T. K. Lau and W. F. Bischof, "Automated detection of breast tumors using the asymmetry approach,' Comput. Biomed. Res., vol. 24, pp.273-295, 1991.

[10] W. P. Kegelmeyer Jr., J. M. Pruneda, P. D. Bourland, A. Hillis, M. W. Riggs, and M. L. Nipper, "Computer-aided mammographic screening for spiculated lesions," Radiol., vol. 191, no. 2, pp. 331-337, May 1994.

[11] D. Marr and E. Hildreth, "Theory of edge detection," in Proceeding Royal Society, London., vol. 207, pp. 187-217, 1980.

[12] J. Lunscher and M. P. Beddoes, "Optimal edge detector design: Parameter selection and noise effects," IEEE Trans. Pattem Anal. Machine Intell., vol. 8, no. 2, pp. 154-176, Mar. 1986.

[13]Duda \& Hart, "Patteren Classification and Scene Analysis", John Wiley and sons, 1973 .

[14]H. B. Kekre, Saylee Gharge, "Segmentation of MRI Images using Probability and Entropy as Statistical parameters for Texture analysis," Advances in Computational sciences and Technology (ACST),Volume 2, No.2, pp: 219-230, 2009, http://www.ripublication.com/acst.htm

[15]H. B. Kekre, Saylee Gharge , "Selection of Window Size for Image Segmentation using Texture Features," International Conference on Advanced Computing \& Communication Technologies (ICACCT-2008) Asia Pacific Institute of Information Technology SD India, Panipat ,08-09 November,2008.
[16]H. B. Kekre, Saylee Gharge, "Image Segmentation of MRI using Texture Features," International Conference on Managing Next Generation Software Applications ,School of Science and Humanities, Karunya University, Coimbatore, Tamilnadu ,05-06 December,2008.

[17]H. B. Kekre, Saylee Gharge , "Statistical Parameters like Probability and Entropy applied to SAR image segmentation," International Journal of Engineering Research \& Industry Applications (IJERIA), Vol.2,No.IV, pp.341-353.

[18]H. B. Kekre , Saylee Gharge , "SAR Image Segmentation using co-occurrence matrix and slope magnitude," ACM International Conference on Advances in Computing, Communication \& Control (ICAC3-2009), pp.: 357-362, 23 24 Jan 2009, Fr. Conceicao Rodrigous College of Engg. Available on ACM portal.

[19]H. B. Kekre, Tanuja K. Sarode ,Saylee Gharge, "Detection and Demarcation of Tumor using Vector Quantization in MRI Images" ,International Journal of Engineering Science and Technology(IJEST), Volume 2,No.2,pp:59-66,2009.

[20]H. B. Kekre, Saylee Gharge, "Tumor Demarcation of Mammography Images Using Entropy with Different Window Sizes", Second International Conference on Emerging Trends in Engineering and Technology, ICETET2009, held at Raisoni College of Engineering, Nagpur, India, pp.: 889-894, 16-18 December 2009. Avaliable at IEEE Xplore.

[21]H. B. Kekre, Tanuja K. Sarode, Saylee Gharge, "Kekre's Fast Codebook Generation Algorithm for Tumor Detection in Mammography Images", International Conference and Workshop on Emerging Trends in Technology (ICWET 2010), at Thakur College of Engineering and Technology (TCET), $26 \& 27$ th February 2010, available on ACM portal .

[22]H. B. Kekre, Tanuja K. Sarode, Saylee Gharge, "Image Segmentation of MRI Images using Vector Quantization Techniques" International Conference on Contours of Computing Technology, 13th-14th March 2010, at Babasaheb Gawade Institute of Technology will be available on Springer LNCS Digital Library.

[23]H. B. Kekre, Tanuja K. Sarode, Saylee Gharge, "Tumor Demarcation in Mammographic Images using Vector Quantization Technique on Entropy Images" International Conference on Contours of Computing Technology, 13th14th March 2010, at Babasaheb Gawade Institute of Technology. Paper will be available on Springer LNCS Digital Library.

[24]Tou, J., and Gonzalez, Pattern Recognition Principles Addison-Wesley Publishing Company 1974.

[25]R. M. Gray, "Vector quantization", IEEE ASSP Mag., pp.: 429, Apr. 1984

[26]Y. Linde, A. Buzo, and R. M. Gray, "An algorithm for vector quantizer design," IEEE Trans.Commun., vol. COM-28, no. 1, pp.: 84-95, 1980

[27]H.B.Kekre, Tanuja K. Sarode, "New Fast Improved Clustering Algorithm for Codebook Generation for Vector 
Quantization", International Conference on Engineering Technologies and Applications in Engineering, Technology and Sciences, Computer Science Department, Saurashtra University, Rajkot, Gujarat. (India), Amoghsiddhi Education Society, Sangli, Maharashtra (India) , 13th - 14th January 2008.

[28]H. B. Kekre, Tanuja K. Sarode, "New Fast Improved Codebook Generation Algorithm for Color Images using Vector Quantization," International Journal of Engineering and Technology, vol.1, No.1, pp.: 67-77, September 2008.

[29]H. B. Kekre, Tanuja K. Sarode, "Fast Codebook Generation Algorithm for Color Images using Vector Quantization," International Journal of Computer Science and Information Technology, Vol. 1, No. 1, pp.: 7-12, Jan 2009.

[30]H. B. Kekre, Tanuja K. Sarode, "An Efficient Fast Algorithm to Generate Codebook for Vector Quantization," First International Conference on Emerging Trends in Engineering and Technology, ICETET-2008, held at Raisoni College of Engineering, Nagpur, India, pp.: 62- 67, 16-18 July 2008. Avaliable at IEEE Xplore.

[31]H. B. Kekre, Tanuja K. Sarode, "Fast Codebook Generation Algorithm for Color Images using Vector Quantization," International Journal of Computer Science and Information Technology, Vol. 1, No. 1, pp.: 7-12, Jan 2009.

[32]H. B. Kekre, Tanuja K. Sarode, "Fast Codevector Search Algorithm for 3-D Vector Quantized Codebook", WASET International Journal of cal Computer Information Science and Engineering (IJCISE), Volume 2, No. 4, pp.: 235-239, Fall 2008. Available: http://www.waset.org/ijcise.

[33]H. B. Kekre, Tanuja K. Sarode, "Fast Codebook Search Algorithm for Vector Quantization using Sorting Technique", ACM International Conference on Advances in Computing, Communication and Control (ICAC3-2009), pp: 317-325, 23-24 Jan 2009, Fr. Conceicao Rodrigous College of Engg., Mumbai. Available on ACM portal.

[34]Jim Z.C. Lai, Yi-Ching Liaw, and Julie Liu, "A fast VQ codebook generation algorithm using codeword displacement", Pattern Recogn. vol. 41, no. 1, pp.: 315-319, 2008.

[35]C.H. Hsieh, J.C. Tsai, Lossless compression of VQ index with search order coding, IEEE Trans. Image Process. vol. 5, No. 11, pp.: 1579-1582, 1996.

[36]Chin-Chen Chang, Wen-Chuan Wu, "Fast Planar-Oriented Ripple Search Algorithm for Hyperspace VQ Codebook", IEEE Transaction on image processing, vol 16, no. 6, pp.: 1538-1547, June 2007.

[37]C. Garcia and G. Tziritas, "Face detection using quantized skin color regions merging and wavelet packet analysis," IEEE Trans. Multimedia, vol. 1, no. 3, pp.: 264-277, Sep. 1999.

[38]H. Y. M. Liao, D. Y. Chen, C. W. Su, and H. R. Tyan, "Real-time event detection and its applications to surveillance systems," in Proc. IEEE Int. Symp. Circuits and Systems, Kos, Greece, pp.: 509-512, May 2006.

[39]J. Zheng and M. Hu, "An anomaly intrusion detection system based on vector quantization," IEICE Trans. Inf. Syst., vol. E89-D, no. 1, pp.: 201-210, Jan. 2006.

[40]H. B. Kekre, Tanuja K. Sarode, Bhakti Raul, "Color Image Segmentation using Kekre's Fast Codebook Generation Algorithm Based on Energy Ordering Concept", ACM International Conference on Advances in Computing, Communication and Control (ICAC3-2009), pp.: 357-362, 23-24 Jan 2009, Fr. Conceicao Rodrigous College of Engg., Mumbai. Available on ACM portal.

[41]H. B. Kekre, Tanuja K. Sarode, Bhakti Raul, "Color Image Segmentation using Kekre's Algorithm for Vector Quantization", International Journal of Computer Science
(IJCS), Vol. 3, No. 4, pp.: 287-292, Fall 2008. Available: http://www.waset.org/ijcs.

[42]H. B. Kekre, Tanuja K. Sarode, Bhakti Raul, "Color Image Segmentation using Vector Quantization Techniques Based on Energy Ordering Concept" International Journal of Computing Science and Communication Technologies (IJCSCT) Volume 1, Issue 2, pp: 164-171, January 2009.

[43]H. B. Kekre, Tanuja K. Sarode, Bhakti Raul, "Color Image Segmentation Using Vector Quantization Techniques", Advances in Engineering Science Sect. C (3), pp.: 35-42, July-September 2008.

[44]H. B. Kekre, Tanuja K. Sarode, "Speech Data Compression using Vector Quantization”, WASET International Journal of Computer and Information Science and Engineering (IJCISE), vol. 2, No. 4, pp.: 251-254, Fall 2008. available: http://www.waset.org/ijcise.

[45]H. B. Kekre, Ms. Tanuja K. Sarode, Sudeep D. Thepade, "Image Retrieval using Color-Texture Features from DCT on VQ Codevectors obtained by Kekre's Fast Codebook Generation", ICGST-International Journal on Graphics, Vision and Image Processing (GVIP), Volume 9, Issue 5, pp.: 1-8, September 2009. Available online at http://www.icgst.com/gvip/Volume9/Issue5/P1150921752.ht $\mathrm{ml}$.

[46]H. B. Kekre, Kamal Shah, Tanuja K. Sarode, Sudeep D. Thepade, "Performance Comparison of Vector Quantization Technique - KFCG with LBG, Existing Transforms and PCA for Face Recognition", International Journal of Information Retrieval (IJIR), Vol. 02, Issue 1, pp.: 64-71, 2009.

[47]L. Vincent, P. Soille, Watersheds in digital spaces: An efficient algorithm based on immersion Simulations, IEEE Trans. PAMI., 13 (6) (1991) 583-593.

[48] F. Meyer, Topographic distance and watershed lines,Signal Processing, 38 (1) (1994) 113-125.

[49] A. Bieniek, A. Moga, An efficient watershed algorithm based on connected components, Pattern Recognition, 33 (6) (2000) 907-916.

[50] M. Frucci, Oversegmentation reduction by flooding regions and digging watershed lines, International Journal of Pattern Recognition and Artificial Intelligence, 20 (2006) 15-38.

[51] L. E. Band, Topographic partition of watersheds with digital elevation models, Water Resources Res., 22 (1) (1986) 1524.

[52]Y. Linde, A. Buzo, and R. M. Gray, "An algorithm for vector quantizer design," IEEE Transactions on Communication., vol. COM-28, pp. 85-94, Jan., 1980.

[53] R. M. Gray, "Vector quantization," IEEE ASSP Magazine, vol. 1, pp. 4-29, 1984.

[54] A. Gersho, and R. M. Gray, Vector quantization and signal compression, Kluwer Academic Publishers, Norwell,1992.

[55]Robert M. Haralick, Statistical and Structural Approaches to Texture, IEEE Proceedings Of vol. 67, no. 5, May 1979.

[56]Leila Shafarenko and Maria Petrou, “ Automatic Watershed Segmentation of Randomly Textured Color Images", IEEE Transactions on Image Processing, Vol.6, No.11, pp.15301544, 1997. 
[57]Basim Alhadidi, Mohammad H. et al, "Mammogram Breast Cancer Edge Detection Using Image Processing Function" Information Technology Journal 6(2):217-221,2007,ISSN$1812-5638$

[58] H. B. Kekre, Tanuja K. Sarode, "2-level Vector Quantization Method for Codebook Design using Kekre's Median Codebook Generation Algorithm", Advances in Computational Sciences and Technology (ACST), ISSN 0973-6107, Volume 2 Number 2, 2009, pp. 167-178. Available online
[59] H. B. Kekre, Tanuja K. Sarode, "Multilevel Vector Quantization Method for Codebook Generation", International Journal of Engineering Research and Industrial Applications (IJERIA), Volume 2, No. V, 2009, ISSN 09741518, pp.: 217-231. Available online at. http://www.ascentjournals.com/ijeria_contents_Vol2No5.htm.

[60] H. B. Kekre, Tanuja K. Sarode "Vector Quantized Codebook Optimization using K-Means", International Journal on Computer Science and Engineering (IJCSE) Vol.1, No. 3, 2009, pp.: 283-290, Available online at: http://journals.indexcopernicus.com/abstracted.php?level=4 \&id issue $=839392$. 